\title{
Evaluación de las características físicas, químicas y sensoriales de la harina de maíz precocidad con salvado de arroz en la elaboración de arepas
}

\author{
Evaluation of the physical, chemical and sensorial characteristics of corn flour \\ precocity with rice bran in the production of arepas
Avaliação das características físicas, químicas e sensoriais da precocidade da farinha de milho com farelo de arroz na produção de arepas

\author{
Meivi Gómez \\ meivigomez19@gmail.com
}

Leonardo Ramírez

joleraca25@gmail.com

Universidad Nacional Experimental de los Llanos Occidentales, “EZEQUIEL ZAMORA”, Venezuela

Artículo recibido octubre 2019, arbitrado noviembre 2019 y publicado en enero 2020

\section{RESUMEN}

El presente artículo muestra la evaluación acerca de las características físicas: expansión, granulometría, así mismo, químicas: humedad y atributos sensoriales: color, sabor y textura de la harina de maíz precocida con salvado de arroz en la elaboración de arepas. Como método fueron elaboradas tres formulaciones de harina de maíz precocida con salvado de arroz de arroz (50:50, 70:30 y 80:20). El análisis de las características físicas demostró que la calidad de las arepas depende significativamente de la expansión y granulometría, ya que se observó que a mayor porcentaje de harina de maíz precocida aumentaba la capacidad de retención de agua, cabe destacar que el salvado de arroz le aporta altos valores nutricionales. Los resultados del análisis sensorial indicaron que entre las tres formulaciones los consumidores se inclinaron por la muestra 493 de harina de maíz precocida con salvado de arroz (80:20) de menor granulometría por lo que se consideró esta mezcla de harina compuesta como la muestra óptima.

Palabras clave: Salvado de arroz; harina de maíz precocida; evaluación física y sensorial

\begin{abstract}
The present article shows the evaluation about the physical characteristics: expansion, grain size, likewise, chemical: humidity and sensorial attributes: color, flavor and texture of the corn flour precooked with rice bran in the preparation of arepas. As a method, three formulations of precooked corn flour with rice bran (50:50, 70:30 and 80:20) were elaborated. The analysis of the physical characteristics showed that the quality of the arepas depends significantly on the expansion and granulomere, since it was observed that the higher the percentage of precooked corn flour the water retention capacity increased, it should be noted that the rice bran provides high nutritional values. The results of the sensory analysis indicated that among the three formulations, consumers favored sample 493 of corn flour precooked with rice bran $(80: 20)$ with a smaller grain size, so this compound flour mixture was considered the optimal sample.
\end{abstract}

Key words: Rice bran; precooked corn flour; physical and sensory evaluation 


\section{RESUMO}

0 presente artigo mostra a avaliação das características físicas: expansão, tamanho do grão e, igualmente, atributos químicos: umidade e sensoriais: cor, sabor e textura da farinha de milho pré-cozida com farelo de arroz no preparo das arepas. Como método, foram elaboradas três formulações de farinha de milho pré-cozida com farelo de arroz (50:50, 70:30 e 80:20). A análise das características físicas mostrou que a qualidade das arepas depende significativamente da expansão e granulometria, uma vez que foi observado que quanto maior o percentual de farinha de milho pré-cozida, maior a capacidade de retenção de água, ressalta-se que o farelo de arroz fornece altos valores nutricionais. Os resultados da análise sensorial indicaram que entre as três formulações, os consumidores preferiram a amostra 493 de farinha de milho précozida com farelo de arroz (80:20) com um tamanho de grão menor, portanto essa mistura de farinha composta foi considerada a amostra ideal.

Palavras-Chave: Farelo de arroz; farinha de milho pré-cozida; avaliação física e sensorial

\section{INTRODUCCIÓN}

El suministro insuficiente de materias primas, como consecuencia de la disminución de la cosecha y la falta de rentabilidad para los actores de la cadena agroproductiva, son los dos problemas que en este momento enfrenta la industria procesadora de harina de maíz precocida en Venezuela. En este sentido, el salvado de arroz es un subproducto que se obtiene a partir de blanquear el arroz integral y representa alrededor del peso del grano, contiene proteína de buena calidad biológica y es además una fuente energética por su contenido en grasa y almidón.

La producción nacional apenas está produciendo el 30\% del maíz blanco que requiere Venezuela, por lo que es urgente acometer acciones inmediatas que permitan la recuperación de la producción, única vía para combatir la escasez y el desabastecimiento y así poder garantizar la soberanía y seguridad alimentaria.
El suministro insuficiente de materias primas, como consecuencia de la disminución de la cosecha y la falta de rentabilidad para los actores de la cadena agroproductiva, son los dos problemas que en este momento enfrenta la industria procesadora de harina de maíz precocida. (Juan B. Salas 2016).

En este sentido, el salvado de arroz es un subproducto que se obtiene a partir de blanquear el arroz integral y representa alrededor del peso del grano, contiene proteína de buena calidad biológica y es además una fuente energética por su contenido en grasa y almidón. Dependiendo de la variedad del cultivo de arroz y del procesado, el salvado de arroz contiene alrededor de $15-20 \%$ de lípidos y $15-16 \%$ de proteínas, $23-28 \%$ de fibra y $7-10 \%$ de cenizas, además presenta un alto contenido de vitaminas del complejo $\mathrm{V}$ y E el e-tocoferol. (Cano, y otros, 2001).

Debido a la gran demanda de harina de maíz precocida, el incremento de los costos de producción y la baja calidad indujo la alternativa de crear un producto innovador como lo es la harina de maíz precocida con salvado de arroz, mezcla en la que el salvado de arroz resulta un ingrediente sumamente importante debido a que en él se encuentra de forma natural un gran número de sustancias funcionales con propiedades antioxidantes, antiinflamatorias, hipolipemiantes y cuando es consumida en bajas proporciones es antidiabética (Martínez y Rodríguez, 2017). Además, el salvado de arroz resulta una materia prima de menor costo que el maíz, por lo que permite reducir los costos de producción. Por consiguiente, nuestro estudio estará enfocado en evaluar las características físicas, químicas y sensoriales de la harina de maíz precocida proveniente de la empresa APARCA C.A ubicada en Venezuela estado Portuguesa mezclada con salvado de arroz. 
En vista de la situación económica actual en que se encuentra el Venezuela, ha afectado considerablemente la calidad de la harina de maíz precocida en la empresa APARCA C.A como consecuencia de la falta de recursos económicos (Valdivieso, 1985).

La relevancia de este trabajo de investigación radica en que se evalúa la propiedades físicas, químicas y sensoriales de un producto con muchísimo potencial y cuyos resultados tienen grandes aplicaciones desde diferentes enfoques como por ejemplo la comercialización de la harina, mejoramiento de otros productos con la harina, así como también ampliar el conocimiento sobre el salvado de arroz, ya que, es un subproducto que no es aprovechado en la industria alimentaria, datos que pueden ser de gran importancia para investigaciones futuras.

\section{MATERIALES Y MÉTODOS}

El presente estudio fue realizado bajo el nivel de una investigación integrativa, según Hurtado (2010). Esta investigación cumple con todas las características, en virtud de que el objetivo general se encuentra dentro del nivel integrativo y tiene como principio evaluar las características físicas, químicas y sensoriales de la mezcla de harina de maíz precocida con salvado de arroz en la elaboración de arepas. La investigación es de tipo experimental en referencia a este fundamento conceptual porque sirvió para para familiarizarse y evaluar el efecto del salvado de arroz sobre las características físicas y químicas de la harina de maíz precocida. (Bruzone, 2014; Baduai, 2006; Rodríguez, 2007). También, es experimental porque puesto a que a través de la variación de la cantidad de salvado de arroz y de harina de maíz pueden proporcionar a las arepas ciertas variaciones que pueden o no originar diferentes resultados en las variables $y$ atributos que se medirán al final del proceso.

Para los materiales se usó la harina de maíz precocida que fue donada por la empresa APARCA C.A, cabe destacar, que el salvado de arroz también fue donado por el comerciante informal Luis Hernández. Se formularon tres muestras utilizando el salvado de arroz de la siguiente forma: 50,70 y $80 \%$.

Tabla 1. Insumos para cada formulación propuesta.

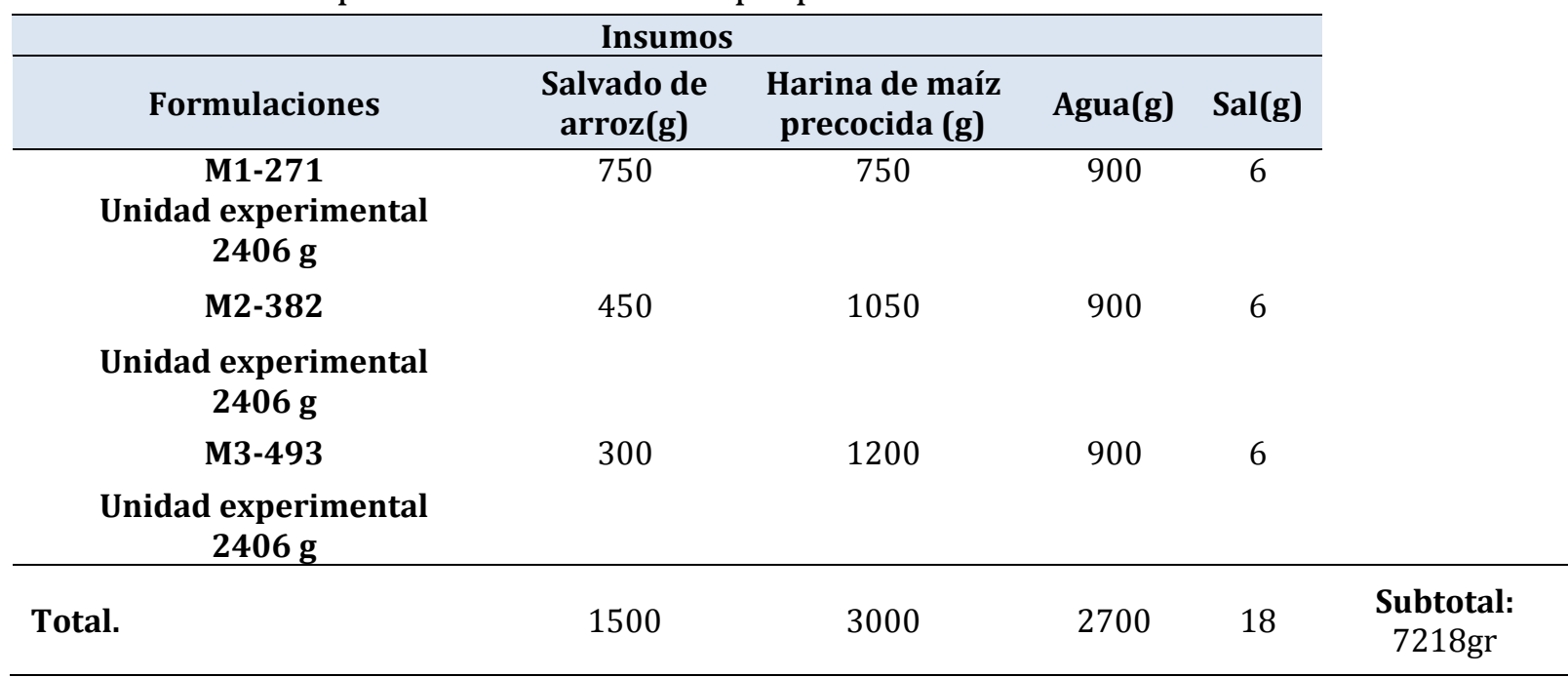




\section{Preparación}

En primer lugar se somete el salvado de arroz a un calentamiento para inactivar las enzimas para así evitar la rancidez hidrolítica. Luego se procede a mezclar con la harina de maíz precocida tomando en consideración que se realizaran tres formulaciones diferentes. Finalmente, se conservará en un espacio a temperatura ambiente $\mathrm{y}$ al transcurrir los días establecidos permitiendo realizar la segunda fase.

\section{Diagrama de proceso de la harina de maíz precocida con salvado de arroz para la elaboración de arepas}

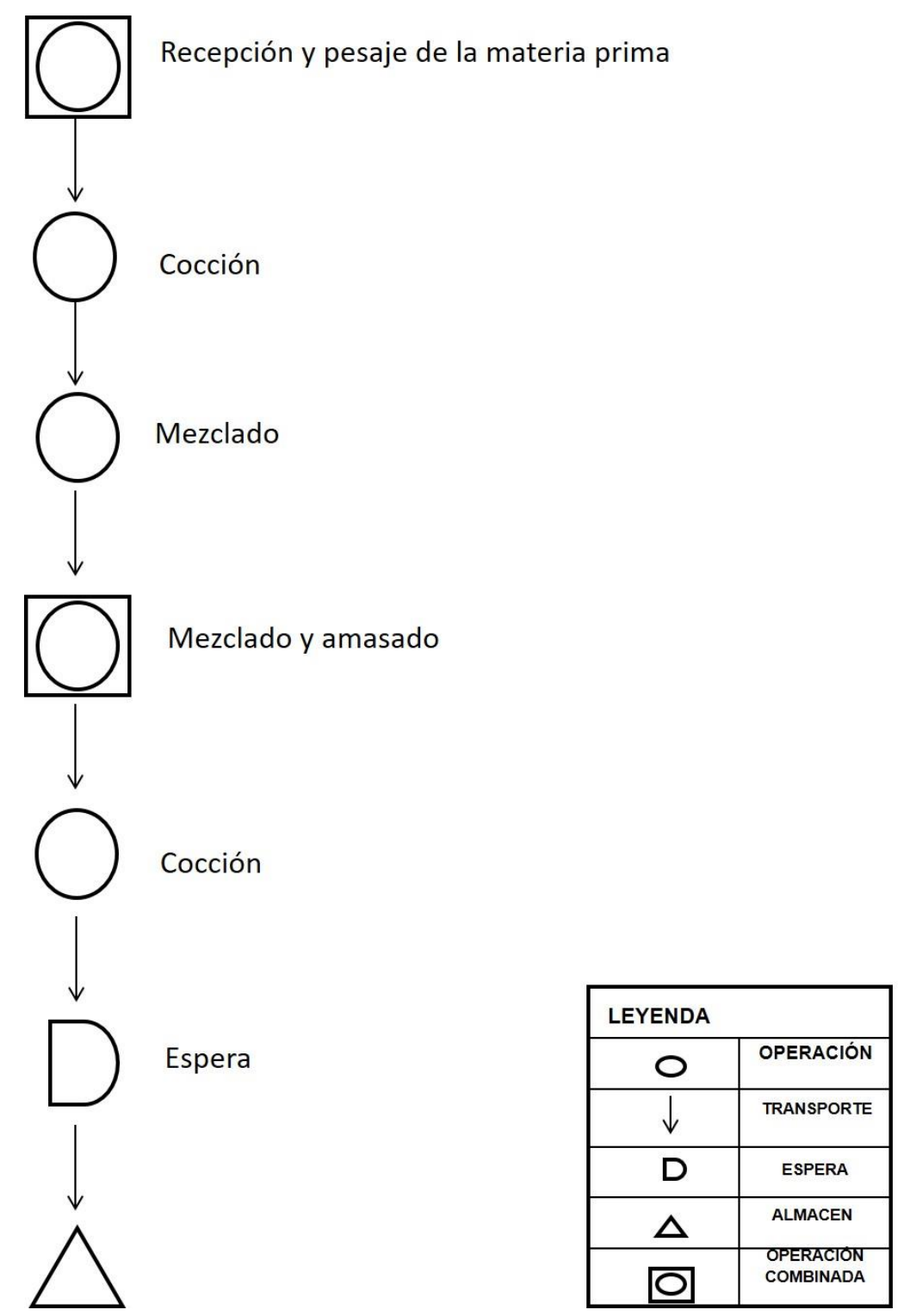

Figura 1. Diagrama de proceso de la harina de maíz precocida con salvado de arroz para la elaboración de arepas. 


\section{Análisis del diagrama de proceso}

Recepción y pesaje: La harina de maíz precocida y el salvado de arroz es previamente obtenidos junto con los demás insumos para desarrollar el producto por esta razón se inspeccionan y pesan atendiendo a las formulaciones (Ver tabla $\mathrm{N}^{\circ} 3$ ) preservando que estén libres de cualquier impureza o sustancia extraña que pueda perjudicar el proceso disminuyendo la calidad del producto final y revisando tarjetas de expedición.

Cocción: Se somete el salvado de arroz a 10 minutos en el microondas donde se inactivaran las enzimas para evitar la rancidez.

Mezclado: Se mescla el salvado de arroz conjuntamente con la harina de maíz precocida procurando que esta sea una mezcla uniforme. Cabe destacar que esto se realizara en diferente formulación ya que son tres muestras. (Torres y Guerra, 2007)
Mezclado y amasado: Se adiciona agua en un envase plástico, así mismo, se le añade sal. Luego se le agrega la harina de maíz precocida con salvado de arroz poco a poco evitando que se formen grumos. Se enfatiza que este proceso es realizado en tres ocasiones puesto a que se realizaran tres muestras para nuestra valoración hedónica.

Cocción: Se procede a realizar las arepas recordando que estas tienen una forma circular, ya lista se pasan al budare por 10 minutos dándole vueltas cada 2 minutos.

Espera: Se dejan reposar durante unos minutos y se envuelven en servilletas.

Almacenado: Se almacenan en cabes que no permiten la entrada de agua. De esta no alterando las atributos sensoriales de las arepas.

\section{Balance de materia}

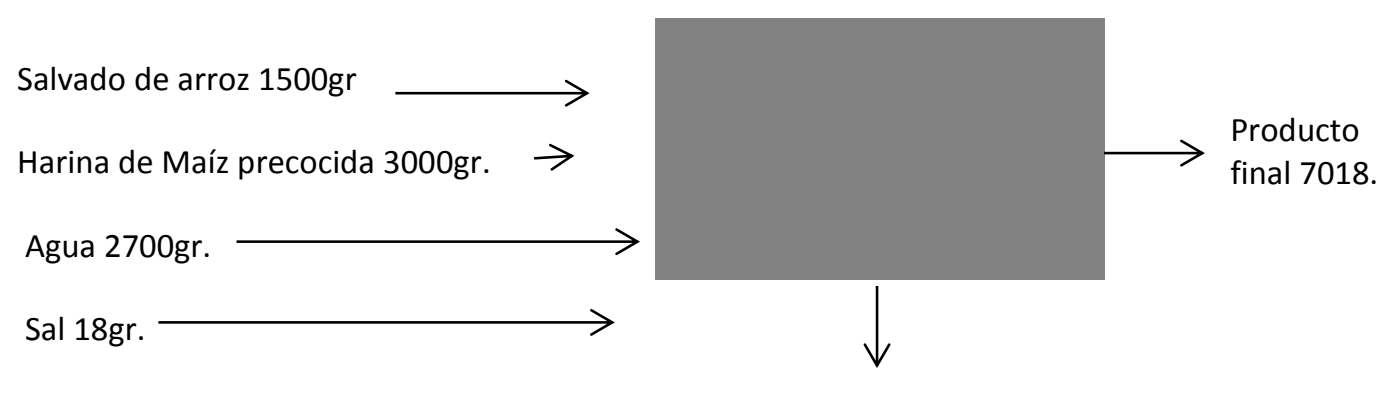

Perdida 200gr

$\mathrm{A}+\mathrm{B}+\mathrm{C}+\mathrm{D}-\mathrm{E}=\mathrm{H}$

$1500 \mathrm{gr}+3000 \mathrm{gr}+2700 \mathrm{gr}+18 \mathrm{gr}-200=7018 \mathrm{gr}$ del producto final. 


\section{Rendimiento}

$\%$ Rendimiento

7218 gr 100

7018 gr X

$\%$ de rendimiento $=\mathbf{9 7 . 2 2} \%$

\section{Pruebas y métodos}

Se hace énfasis, ya que estos análisis se realizan para tres muestras de la harina de maíz precocida con salvado de arroz. Las cual des damos por nombre M1 271, M2 382 y M2 493. (Hernández, Guerra y Rivero 1999).

Estos análisis se realizaron en la empresa Almacenadora Paso Real C.A ubicada, en la República Bolivariana de Venezuela en el municipio Guanarito estado Portuguesa.

\section{Equipos e instrumentos a utilizar}

- Vaso de precipitado de $1000 \mathrm{ml}$.

- Espátula.

- Bata.

- Gorro.

- Tapa boca.

- Papel calibrado con círculos concéntricos desde 7,28 cm hasta 35,28 cm. Aumentando de centímetro en centímetro.

- Cilindro de acero inoxidable de 7,28 de diámetro interno por 7,62 cm de alto.

- Nivel para ajustar la mesa de vidrio.

- Platillo de aluminio de diámetro interno de $12 \mathrm{~cm}$ especial para la balanza

- Balanza OHAUS

- Equipo cernidor marca roptap.

- Determinador de humedad marco OHAUS.

- Mesa de vidrio de expansión de $40 \mathrm{~cm} \mathrm{x}$ $40 \mathrm{~cm}$.

Granulometría: Se pesan 100gramos de la muestra en una balanza, luego se procede a graduar el equipo cernidor de marca roptap por 10 minutos. Transcurrido los 10 minutos se procede a pesar el contenido que haya quedado en los tamices cabe destacar que se expresa directamente en porcentajes.

Humedad: Se gradúa la balanza de humedad para harina de maíz precocida, se verifica que el equipo este limpio. Con una espátula se va agregando lentamente la muestra hasta llegar a 3 gramos de la muestra, cierra la tapa y pulsa Star, se esperan 4 minutos y se lee el porcentaje de humedad.

Expansión: De acuerdo a los datos obtenidos por humedad, se busca en la tabla establecida por COVENIN la cantidad de agua y harina a mezclar. En un vaso precipitado se agrega la harina requerida y el gua mezclando al mismo tiempo para evitar la formación de grumos, se continúa mezclando durante tres minutos y se deja reposar por unos minutos.

Se coloca el cilindro en el centro del grafico sobre el circulo correspondiente, transcurrido el tiempo de reposo se le dan 10 vueltas a la mezcla se deposita en el cilindro el cual se llena completamente y se enrasan con la espátula, al transcurrir el minuto se levanta el cilindro suavemente. Se toman 8 lecturas sobre cada radio dibujado y se saca el promedio como valor final.

Para llevar a cabo esta fase del estudio se procedió a elaborar las muestras experimentales y análisis de la variable de respuesta.

\section{RESULTADOS Y DISCUSIÓN}

El siguiente apartado muestra los resultados alcanzado con la evaluación de las características físicas, químicas y sensoriales de la harina de maíz precocidad con salvado de arroz en la elaboración de arepas, obtenido en la recolección de datos. 


\section{Evaluación física y química}

La tabla 2 se muestra los resultados en cuanto a las valoraciones de la harina de maíz precocida con salvado de arroz tomando como parámetros la expansión, humedad y granulometría de tres muestras aleatorias, las mismas se formularon utilizando el salvado de arroz de la siguiente forma: 50, 70 y $80 \%$.

Tabla 2. Valoraciones de la harina de maíz precocida con salvado de arroz para humedad y expansión

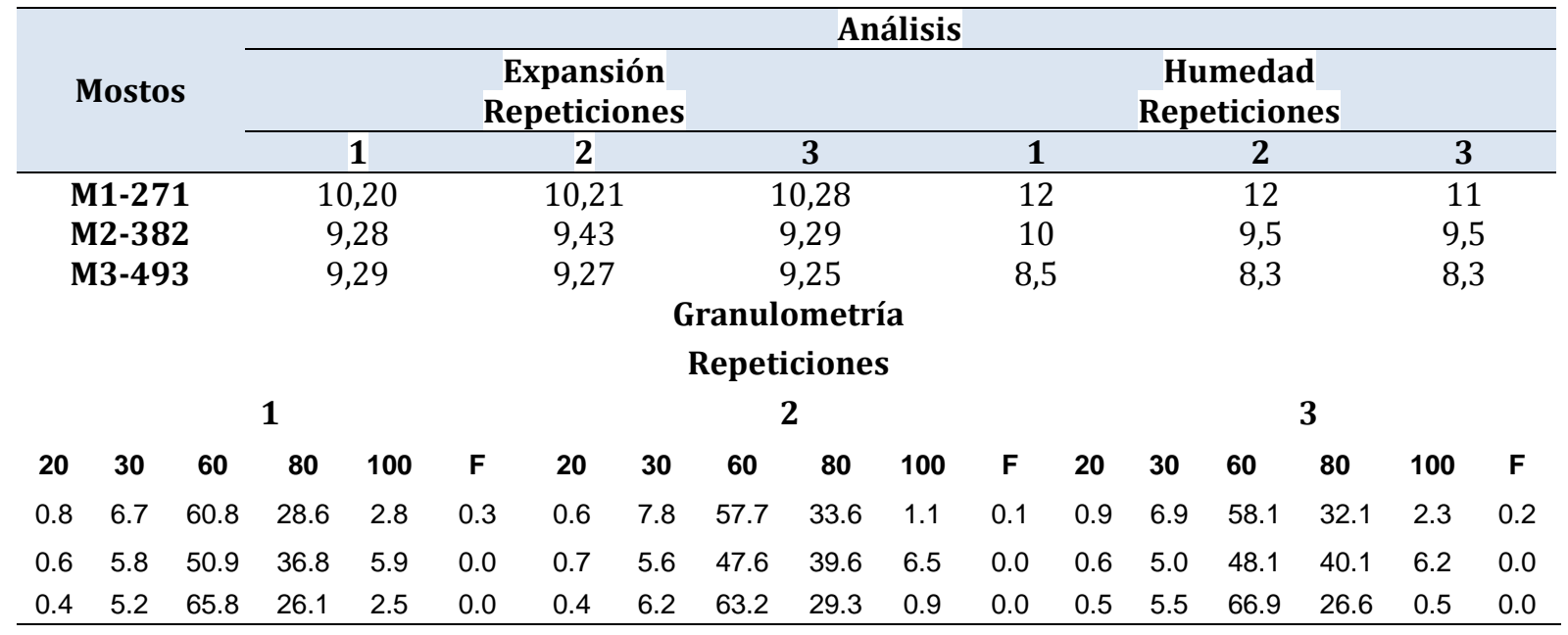

Efectos físicos y químicos obtenidos mediante la aplicación del análisis estadístico SPSS

Tabla 3. Resultados físicos y químicos obtenidos mediante la aplicación del análisis estadístico SPSS.

\begin{tabular}{clc}
\hline Características & \multicolumn{1}{c}{ Muestras } & Media \pm Desviación estand. \\
\hline \multirow{3}{*}{ Humedad. } & M1: 50-50 código 271 & $10,16 \pm 1,755$ \\
& M2: 70-30 & $9,93 \pm 1,888$ \\
& M3: 80-20 código 493 & $9,56 \pm 1,401$ \\
& M1: 50-50 código 271 & $9,59 \pm, 5283$ \\
Expansión. & M2: 70-30 & $9,63 \pm, 5029$ \\
& Código 382 & $9,60 \pm, 5834$ \\
\hline
\end{tabular}

Estas valoraciones fueron tratadas en el SPSS v. 24; para estimar correlación entre los factores estudiados arrojando respectivamente valores de los supuestos que deben anteceder para aplicar ANOVA, siendo necesario realizar una tabla que resume los estadísticos más relevantes para reconocer si se acepta o rechaza la hipótesis nula. (Tabla 3). 
En cuento, para los análisis obtenidos para humedad se puede concluir que existen suficientes evidencias para aceptar la hipótesis nula, es decir, la cantidad del salvado de arroz no influye en las características de humedad de la harina de maíz precocida. Se puede observar el valor de la desviación estándar el cual es menor que dos.

Al agregarle salvado de arroz aumenta la humedad en la muestra, tal es el caso de la muestra 271 y 382.

En atención a los resultados se puede concluir que existen suficientes evidencias para aceptar la hipótesis nula, es decir, la cantidad del salvado de arroz no influye en las características de expansión de la harina de maíz precocida. Se puede observar el valor de la desviación estándar el cual fue menor que dos.

\section{Granulometría}

Debido, a que para el análisis de los datos de granulometría es muy amplio, se decidió hacer por separados.

En atención a los resultados se puede concluir que existen suficientes evidencias para rechazar la hipótesis nula, es decir, la cantidad del salvado de arroz si influye en las características físicas para el análisis de granulometría para la harina de maíz precocida. Se puede observar el valor de la desviación estándar el cual es mayor que dos. (Tabla 4).

Tabla 4. Resultados obtenidos en estadística descriptiva para granulometría

\begin{tabular}{lcccccc} 
& N & Mínimo & Máximo & Media & $\begin{array}{c}\text { Desviación } \\
\text { estándar }\end{array}$ & Varianza \\
\hline Granulometría & 45 &, 00 & 66,90 & 19,3800 & 23,10983 & 534,064 \\
N válido (por lista) & 45 & & & & & \\
\hline
\end{tabular}

\section{Evaluación sensorial}

Desde la información recaudada por la aplicación de la escala hedónica se tienen a realizar los cuadros con los siguientes descriptores:

1: Me Disgusta Mucho

2: Me Disgusta Moderadamente

3: Me Disgusta Levemente

4: No Me Gusta Ni Me Disgusta

5: Me Gusta Levemente

6: Me Gusta Moderadamente

7: Me Gusta Mucho 
Tabla 5. Aplicación del análisis sensorial

\begin{tabular}{cccc}
\hline Descriptores & $\begin{array}{c}\text { M1: 50-50 } \\
\text { código 271 }\end{array}$ & $\begin{array}{c}\text { M2: 70-30 } \\
\text { código 382 }\end{array}$ & $\begin{array}{c}\text { M3: 80-20 } \\
\text { código 493 }\end{array}$ \\
\hline 1 & 0 & 0 & 0 \\
2 & 0 & 0 & 0 \\
3 & 2 & 3 & 0 \\
4 & 4 & 4 & 7 \\
5 & 21 & 14 & 8 \\
6 & 17 & 27 & 18 \\
7 & 26 & 22 & 37 \\
\hline Totales: & $\mathbf{7 0}$ & $\mathbf{7 0}$ & $\mathbf{7 0}$
\end{tabular}

En la tabla 5 se presentan los resultados obtenidos mediante la aplicación de la encuesta, de esta forma se demuestra que la muestra con mayor aceptación fue la 493, seguida de la muestra 382 quedando en último lugar la muestra 271.

\section{Análisis estadístico de kruskal-Wallis a nivel general para los atributos sensoriales}

Tabla 6. Resultados obtenidos mediante Kruskal-Wallis a nivel general de los atributos sensoriales.

\begin{tabular}{cccc}
\hline Atributo sensorial. & Color & Sabor & Textura \\
\hline Sig. Asintótica &, 000 &, 000 &, 000 \\
\hline
\end{tabular}

En atención a los resultados se puede concluir que existen suficientes evidencias para aceptar la hipótesis nula, es decir, la cantidad de salvado de arroz no influye en las características sensoriales de la harina de maíz precocida para las tres muestras en estudio, se puede observar que el valor sig $=0,000$ el cual es menor que el nivel de significancia 0,05 . (Tabla 6).
Diferencias significativas entre las muestras mediante la aplicación del análisis estadístico SPSS aplicando la prueba Duncan 271 y 382

Aplicando Duncan mediante el análisis estadístico SPSS podemos confirmar que las muestras 271 y 382 presentan diferencias significativas, se pueden observar los valores distribuidos individualmente en cada columna del uno al cinco. (Tabla 7).

Tabla 7. Diferencias significativas entre las muestras 271 y 382.

\begin{tabular}{ccccccrr}
\hline \multirow{2}{*}{ Muestra 382 } & $\mathbf{N}$ & \multicolumn{6}{c}{ Subconjunto para alfa = 0.05 } \\
\cline { 2 - 7 } & 3 & 1,3333 & $\mathbf{2}$ & $\mathbf{3}$ & $\mathbf{4}$ & $\mathbf{5}$ \\
\hline $\mathbf{3 , 0 0}$ & 4 & & 3,0000 & & & \\
$\mathbf{4 , 0 0}$ & 14 & & & 3,7143 & & \\
$\mathbf{5 , 0 0}$ & 27 & & & & 4,9630 & \\
$\mathbf{6 , 0 0}$ & 22 & & & & & 5,9545 \\
$\mathbf{7 , 0 0}$ & & 1,000 & 1,000 & 1,000 & 1,000 & 1,000 \\
Sig. & & & & & & & \\
\hline
\end{tabular}


Diferencias significativas entre las muestras mediante la aplicación del análisis estadístico SPSS aplicando la prueba Dunca entre la muestra 382 y 493

En este sentido, también podemos confirmar que las muestras dos (70-30) y tres
(80-20) presentan diferencias significativas, se pueden observar los valores distribuidos individualmente en cada columna del dos al cuatro. Sin embargo, para la primera columna las categorías 3 y 4 aparecen en la misma por lo que entre este par no hay diferencias significativas. (Tabla 9).

Tabla 8. Diferencias significativas para la muestra 382 y 493

\begin{tabular}{cccccc}
\hline Muestra 382 & $\mathbf{N}$ & \multicolumn{5}{c}{ Subconjunto para alfa $=\mathbf{0 . 0 5}$} \\
\cline { 3 - 6 } & & $\mathbf{1}$ & $\mathbf{2}$ & $\mathbf{3}$ & $\mathbf{4}$ \\
\hline $\mathbf{3 , 0 0}$ & 4 & 4,0000 & & \\
$\mathbf{4 , 0 0}$ & 14 & 4,0000 & & & \\
$\mathbf{5 , 0 0}$ & 27 & & 5,4286 & & \\
$\mathbf{6 , 0 0}$ & 22 & & & 6,5556 & \\
$\mathbf{7 , 0 0}$ & & 1,000 & 1,000 & 1,000 & 1,000 \\
$\mathbf{S i g}$ & & & & & \\
\hline
\end{tabular}

\section{Análisis estadístico para color}

En atención a los resultados se puede concluir que existen suficientes evidencias para aceptar la hipótesis nula, es decir, existen diferencias significativas en cuanto al color de las arepas elaboradas con las distintas concentraciones 50-50, 70-30, 80-20. Se puede observar el valor sig=0,000 que es un valor menor que el nivel de significancia 0,05. (Tabla 9).

Tabla 9. Prueba de Kruskal-Wallis para atributos de color.

\begin{tabular}{cccc}
\hline Muestra & $\mathbf{2 7 1}$ & $\mathbf{3 8 2}$ & $\mathbf{4 9 3}$ \\
\hline Chi-cuadrado & 69,000 & 69,000 & 69,000 \\
Sig. Asintótica &, 000 &, 000 &, 000 \\
\hline
\end{tabular}

Los degustadores no encontraron diferencia en ninguna de las muestras a diferentes formulaciones.

\section{Análisis estadístico para sabor}

En atención a los resultados se puede concluir que existen suficientes evidencias para aceptar la hipótesis nula, es decir, no existen diferencias significativas en cuanto al sabor de las arepas elaboradas con las distintas concentraciones 50-50, 70-30, 80-20. Se puede observar el valor sig=0,000 que es un valor menor que el nivel de significancia 0,05 . (Tabla 10) 
Tabla 10. Resultados de la prueba Kruskal Wallis para sabor

\begin{tabular}{cccc}
\hline Muestra & $\mathbf{2 7 1}$ & $\mathbf{3 8 2}$ & $\mathbf{4 9 3}$ \\
\hline Chi-cuadrado. & 52,720 & 69,000 & 69,000 \\
Sig. Asintótica. &, 000 &, 000 &, 000 \\
\hline
\end{tabular}

Según los datos obtenidos mediante la aplicación de la encuesta no hay una diferencia significativa entre las muestras 382 y 493 ya que no hubo una gran variación estos son demostrados en la tabla 10.

\section{Análisis estadístico para textura}

En atención a los resultados se puede concluir que existen suficientes evidencias para aceptar la hipótesis nula, es decir, la cantidad de salvado de arroz si influye en cuanto a la textura de las arepas elaboradas con las distintas concentraciones para las tres muestras en estudio, se puede observar que el valor sig=0,000 el cual es menor que el nivel de significancia 0,05. (Tabla 11).

Tabla 11. Análisis estadístico Kruskal-Wallis

\begin{tabular}{lccc}
\hline Muestra & $\mathbf{2 7 1}$ & $\mathbf{3 8 2}$ & $\mathbf{4 9 7}$ \\
\hline Chi-cuadrado. & 52,720 & 52,257 & 59,866 \\
Sig. Asintótica &, 000 &, 000 &, 000 \\
\hline
\end{tabular}

Diferencias significativas entre las muestras mediante la aplicación del análisis estadístico SPSS aplicando la prueba Dunca entre la muestra 271 y 382

En este sentido, también podemos confirmar que las muestras uno (50-50) y dos
(70-30) presentan diferencias significativas, se pueden observar los valores distribuidos individualmente en cada columna del dos al tres. Sin embargo, para la primera columna las categorías 3, 4 y 5 aparecen en la misma por lo que entre este trio no hay diferencias significativas. (Tabla 12).

Tabla 12. Diferencias Significativas entre la muestra 271 y 382

\begin{tabular}{ccccc}
\hline \multirow{2}{*}{ Muestra 382 } & $\mathbf{N}$ & \multicolumn{3}{c}{ Subconjunto para alfa $=\mathbf{0 . 0 5}$} \\
\cline { 3 - 5 } & & $\mathbf{1}$ & $\mathbf{2}$ & $\mathbf{3}$ \\
\hline $\mathbf{3 , 0 0}$ & 2 & 1,5000 & & \\
$\mathbf{4 , 0 0}$ & 4 & 2,0000 & & \\
$\mathbf{5 , 0 0}$ & 21 & 2,1429 & & \\
$\mathbf{6 , 0 0}$ & 17 & & 3,0000 & \\
$\mathbf{7 , 0 0}$ & 26 & & & 1,000 \\
Sig. & &, 131 & 1,000 & \\
\hline
\end{tabular}




\section{Diferencias significativas entre las muestras mediante la aplicación del análisis estadístico SPSS aplicando la prueba Dunca entre la muestra 382 y 493}

En este sentido, también podemos confirmar que las muestras dos (70-30) y tres (80-20) presentan diferencias significativas, se pueden observar los valores distribuidos individualmente en cada columna del uno al cinco.

Tabla 13. Diferencias Significativas entre la muestra 271 y 382

\begin{tabular}{ccccccc}
\hline Muestra 493 & \multirow{N}{*}{$\mathbf{5}$} & \multicolumn{5}{c}{ Subconjunto para alfa $=\mathbf{0 . 0 5}$} \\
\cline { 3 - 6 } & & $\mathbf{1}$ & $\mathbf{2}$ & $\mathbf{3}$ & $\mathbf{4}$ & $\mathbf{5}$ \\
$\mathbf{3 , 0 0}$ & 2 & 4,0000 & & & & \\
$\mathbf{4 , 0 0}$ & 4 & & 4,7500 & & \\
$\mathbf{5 , 0 0}$ & 21 & & & 5,4762 & & \\
$\mathbf{6 , 0 0}$ & 17 & & & 6,0000 & \\
$\mathbf{7 , 0 0}$ & 26 & & & & & 6,9615 \\
Sig. & & 1,000 & 1,000 & 1,000 & 1,000 & 1,000 \\
\hline
\end{tabular}

La muestra de 493 obtuvo mayor puntuación en cuanto a textura, seguida de la muestra de 382. Tomando como referencia las proporciones en las que se adiciono salvado de arroz, se determinó que a menor porcentaje mayor será la aceptación.

\section{Discusión y Análisis de la evaluación y las características química y física de la arepa de maíz precocida}

Para las características químicas se obtuvo una aceptación, de esta forma, se cumplió la hipótesis nula, además se debe constatar que en la investigación realizada por Pacheco y Peña, se obtuvieron valores diferentes, debido a que ellos realizaron este análisis en las arepas. De acuerdo a lo antes mencionados, le proporción en la que adicionaron salvado de arroz fue menor. En ese sentido, se destaca que a mayores proporciones de salvado de arroz mayor será la humedad, debido a que no se le aplica un proceso que permita disminuir su actividad de agua, ya que en él se encuentra un sin número de nutrientes.
Por otra parte, para los análisis físicos como expansión, se debe disminuir el porcentaje de salvado de arroz, la muestra 271 si tuvo diferencias significativas en comparación de las muestras 382 y 493, ya que, varían las formulaciones. Para obtener valores mayores se debe aplicar un proceso de precocción que permita la desorganización del almidón, permitiendo que la masa pueda absorber mayores porcentajes de agua.

Para granulometría se tiene que las partículas de menor grosor son las de salvado de arroz, es decir que si influye considerablemente. Además esta facilita el amasado para la posterior elaboración arepas, así mismo, aumenta la viscosidad atrapando aquellas partículas de aguas.

Siguiendo el mismo orden de ideas, en la investigación de Pacheco y Peña, para efectos de la primera evaluación sensorial se tomaron en cuenta las cuatro formulaciones seleccionadas. Se detectó en cuanto al grado de aceptabilidad, en la primera evaluación que las arepas de maíz 100\% con 5\% de salvado de arroz fueron las más aceptadas y la 
arepa con la mayor proporción de salvado de arroz (15\%) fue la de menor preferencia especialmente por el color, siendo esta la principal razón de su descarte en la siguiente evaluación, al parecer el exceso de salvado de arroz en la formulación le confiere un color más oscuro a las arepas, a pesar de que le da mayor suavidad. Estos, valores se asemejan a los obtenidos, debido a que en la muestra 271 había mayor porcentaje de salvado de arroz, en comparación con las muestras 372 y 493 que obtuvieron mayor aceptabilidad. Cabe destacar que no hubo una variación significativa entre todas las muestras, ya que, todas presentaban un color oscuro, por lo que se tiende a rechazar.

\section{CONCLUSIONES}

El salvado de arroz no influyo significativamente sobre las características químicas, valores que se mostraron mediante la aplicación del análisis estadístico. Cabe señalar, que según los resultados obtenidos a mayor cantidad de salvado de arroz, mayor será la humedad.

En cuanto a las características físicas se tiene que para el análisis de expansión, no causo mayor influencia para la muestra 382 y 493 debido a que presentaba mayor porcentaje de harina de maíz precocida, debido a que esta permite la retención de agua, de igual forma, no son significativos los porcentajes de variación. En este mismo sentido, las muestras 271 presentan menores porcentajes de retención de agua, ya que, el salvado de arroz no fue pasado por un proceso de precocción que permita el desdoblamiento del almidón.

Por otra parte, para el análisis de granulometría se tiene que, según el análisis estadístico, se rechaza la hipótesis nula es decir, el grosor de las partículas si es influenciado, debido a que el salvado de arroz es obtenido de la capa externa del arroz, es decir, de la aleurona.
El análisis sensorial permitió cuantificar satisfactoriamente el promedio de rangos de media de los atributos sensoriales sabor, color y textura. Obteniendo que la muestra 493 y 372 presenta la mayor aceptación en el atributo color, sabor y textura. La muestra 493 presenta la mayor aceptación en los atributos sensoriales seguida de la muestra 383.

Basándonos en los resultados obtenidos se puede llegar a la conclusión que el salvado de arroz puede ser utilizado en la agroindustria de harina de maíz precocida, ya que, le aporta alto valores nutricionales que no posee. Además, se disminuye costos de producción porque es un subproducto que no está siendo aprovechado.

\section{REFERENCIAS}

Baduai, S. (2006). Química de los alimentos. Cuarta Edición Pearson Educación, México.

Bruzone, F. (2014) Aplicación de metodología de caracterización sensorial con consumidores de productos lácteos funcionales

Cano, O; Tosquy, OH; Sierra, M; Rodríguez, FA. (2001). Fertilización y densidad de población en genotipos de maíz cultivados bajo condiciones de temporal. Agronomía Mesoamericana 12(2):193-197

Hernández, Guerra y Rivero (1999) Harinas compuesta de endospermo germen de maíz y su uso en la preparación de maíz

Hurtado, J. (2010). Metodología de la Investigación. Guía para la comprensión holística de la ciencia, 4, 411-834

Martínez y Rodríguez. (2017). Evaluación de las propiedades físicas y sensoriales de un pan tipo campesino a base de batata enriquecida con chia

Pacheco, E y Peña, J. (2006) Analizar el efecto de salvado de arroz sobre los parámetros químicos, físicos y sensoriales de arepas precocidas congeladas

Rodríguez, M. (2007) Determinación de la composición química y propiedades físicas del pulido de arroz (Oriza Sativa L.) 
Torres y Guerra. (2007) Elaboración de una harina de quinchoncho (Cajanus Cajan) procesado y mezclado con harina de maíz precocida a diferentes concentraciones para la elaboración de arepas
Valdivieso, R. (1985). Estudio de un caso de innovación tecnológica desarrollada por una empresa venezolana: la harina precocida de maíz. Fundación CIEPE, Vol. 5 (1) 1 